\title{
ABUNDANCE RATIOS IN METAL-POOR GLOBULAR CLUSTERS: DEEP MIXING AND ITS EFFECT ON STELLAR POPULATIONS OF THE GALACTIC HALO
}

\author{
ROBERT P. KRAFT \\ UCO/Lick Observatory \\ University of California, Santa Cruz, CA 95064
}

\section{Primordial vs Evolutionary Scenarios}

Only a bit more than 25 years ago, it seemed possible to assume that all Galactic globular clusters were chemically homogeneous. There were indications that star-to-star Fe abundance variations existed in $\omega$ Cen, but this massive cluster appeared to be unique. Following Osborn's (1971) initial discovery, Zinn's (1973) observation that M92 asymptotic giant branch (AGB) stars had weaker G-bands than subgiants with equivalent temperatures provided the first extensive evidence that there might be variations in the abundances of the light elements in an otherwise "normal" cluster. Since then star-to-star variations in the abundances of $\mathrm{C}, \mathrm{N}, \mathrm{O}, \mathrm{Na}, \mathrm{Mg}$ and $\mathrm{Al}$ have been observed in all cases in which sample sizes have exceeded 5-10 stars, e.g., in clusters such as M92, M15, M13, M3, $\omega$ Cen, M10 and M5. Among giants in these clusters one finds large surface O abundance differences, and these are intimately related to differences of other light element abundances, not only of $\mathrm{C}$ and $\mathrm{N}$, but also of $\mathrm{Na}, \mathrm{Mg}$ and $\mathrm{Al}$ (cf. reviews by Suntzeff 1993, Briley et al 1994, and Kraft 1994). The abundances of $\mathrm{Na}$ and $\mathrm{O}$, as well as $\mathrm{Al}$ and $\mathrm{Mg}$, are anticorrelated. Prime examples are found among giants in M15 (Sneden et al 1997), M13 (Pilachowski et al 1996; Shetrone 1996a,b; and Kraft et al 1997) and $\omega$ Cen (Norris \& Da Costa 1995a,b).

These observed anticorrelations almost certainly result from proton- capture chains that convert $\mathrm{C}$ to $\mathrm{N}, \mathrm{O}$ to $\mathrm{N}, \mathrm{Ne}$ to $\mathrm{Na}$ and $\mathrm{Mg}$ to $\mathrm{Al}$ in or near the hydrogen fusion layers of evolved cluster stars. But which stars? An appealing idea is that during the giant branch lifetimes of the low-mass stars that we now observe, substantial portions of the stellar envelopes have been cycled through regions near the H-burning shell where proton-capture nucleosynthesis can occur. This so-called "evolutionary" scenario involving deep envelope mixing in first ascent red giant branch (RGB) stars has been studied by Denissenkov \& Denissenkova (1990), Langer \& Hoffman (1995), Cavallo et al (1996, 1997) and Langer et al (1997). The mixing mechanism that brings proton-capture products to the surface is poorly understood (Denissenkov \& Weiss 1996, Denissenkov et al 1997, Langer et al 1997), but deep mixing driven by angular momentum has been suggested (Sweigart \& Mengel 1979, Kraft 1994, Langer \& Hoffman 1995, Sweigart 1997). Arguments in favor of the evolutionary picture include the constancy of $\mathrm{C}+\mathrm{N}+\mathrm{O}$ in M13 (Brown et al 1991, Smith et al 1996) and M15 (Sneden et al 1997), the constancy of $\mathrm{Mg}+\mathrm{Al}$ in M13 (Shetrone 1996a), the steady average decline of $\mathrm{C}$ with evolutionary state in M92 (Carbon et al 1982, Langer et al 1986), M15 (Trefzger et al 1983), and NGC 6397 (Briley et al 1990), and the shift toward higher Na abundances with advancing evolutionary state in M13 (Pilachowski et al 1996, Kraft et al 1997).

On the other hand, the so-called "primordial" scenario attributes these required abundance redistributions to a preceding generation of higher mass cluster stars, whose interiors can more easily attain the very high temperatures needed to sustain advanced proton-capture syntheses. Several lines of evidence suggest the need for a primordial scenario. For example, M5 giants that have high $\mathrm{O}$ abundances (Sneden et al 1992) in some instances also have high $\mathrm{N}$ abundances (Briley \& Smith 1993, Smith et al 1997), which suggests that there are primordial variations of $N$. Even more strikingly, there are strong indications that the main sequence stars in 47 Tuc exhibit a positive correlation between $\mathrm{N}$ and $\mathrm{Na}$ (Briley et al 1996). It is hard to understand how deep mixing could provide a mechanism for altering surface composition among main sequence stars. Finally, M15 giants exhibit a bi-modal distribution of Eu (Sneden et al 1997), an element that surely was 
synthesized in a high temperature Type II supernova environment, and which could not have been altered by deep mixing in low-mass red giants. (Interestingly, these Eu variations are completely unrelated to the variations of $\mathrm{O}, \mathrm{N}, \mathrm{Na}, \mathrm{Mg}$ and $\mathrm{Al}$ also seen in this cluster).

The Sweigart \& Mengel (1979) mixing theory predicts that the efficiency of deep mixing should increase with decreasing metallicity. Observations of carbon isotope ratios (Briley et al 1994) and of $\mathrm{Na}$ and $\mathrm{O}$ abundances of red giants in relatively metal-rich clusters such as M71 (Sneden et al 1994) tend to support this idea (cf Bell et al 1979, Dickens et al 1979, Bell \& Dickens 1980). Considered together, the observations currently suggest that all clusters begin with primordial variations in at least some proton capture elements, and that these variations are modified or enhanced by deep mixing in the giant star stage of evolution, and that the degree of modification in general increases with decreasing metallicity. Thus the "debate" between the validity of primordial and evolutionary scenarios may be no debate at all. Both scenarios are needed to explain what we see in the spectra of globular cluster stars.

\section{Deep Mixing and the Second Parameter Problem}

The "second parameter problem" arises from the observation that the morphology of the horizontal branch (HB) in a cluster color-magnitude diagram can vary among clusters having the same Fepeak metallicity (Sandage \& Wildey 1967, van den Bergh 1967). The triplet of clusters M3, M13 and NGC7006 provides one of the best examples: they have Fe-peak metallicities $([\mathrm{Fe} / \mathrm{H}] \sim-1.6)$ differing by less than 0.1 dex (Kraft et al 1993, Smith et al 1997), and although their RGB sequences are quite similar, their HB morphologies are distinctly different. The HB of M3 is populated moreor-less uniformly from $(B-V) \sim-0.2$ all the way to the RGB, and contains nearly 200 RR Lyraes. The HB of M13, on the other hand has no stars redward of the RR Lyrae domain and almost no RR Lyraes; its HB is confined to stars lying blueward of the instability strip. The HB of NGC7006 is opposite to that of $\mathrm{M} 13$, in that a large fraction of its $\mathrm{HB}$ population lies redward of the RR Lyraes. Another factor besides metallicity must determine the HB morphology, and this unknown factor has come to be called the "second parameter".

Following several years of discussion and debate, cluster age has emerged as the most likely factor controlling the second parameter (Searle \& Zinn 1978; Preston et al 1991; Lee et al 1988,1994; Zinn 1993). If age differences between globular clusters provide the correct explanation, important consequences follow for the history of the Galaxy, since the clusters having "excessively" red HBs for their metallicity are preferentially found in the outer Galactic halo. This in turn implies that the age of the halo increases with decreasing Galactocentric distance.

Whether age is the unique driver of the second parameter effect has not been determined. The outer halo clusters Pal 12, Rup 106, Arp 2 and Ter 7 are almost certainly 3-4 Gyr younger than typical Galactic globular clusters (Gratton \& Ortolani 1988, Stetson et al 1989, Buonanno et al 1990, $\mathrm{Da}$ Costa et al 1992). However, these four anomalously young clusters have kinematics associated with the Magellanic Stream, and may therefore be objects in the process of being accreted to the Galaxy. As for the other halo clusters, Richer et al (1996) have considered 35 for which there are well-established deep CCD-based color-magnitude arrays, and have concluded that there is no compelling evidence for an age gradient or for age being the unique driver of the second parameter. Similar conclusions have been reached also by Stetson et al (1996).

If age is not entirely responsible for the second parameter effect, what else is plausible as an explanation? High resolution spectroscopic studies have revealed an interesting distinction between the giants in the "blue HB" clusters M10 and M13 (Kraft et al 1992, 1993, 1995), the "normal HB" cluster M3 (Kraft et al 1992), and the "red HB" cluster NGC7006 (Smith et al 1997). First-ascent RGB stars in M10 and especially in M13, in contrast with M3 and especially NGC7006 giants in the same evolutionary stage, show far more evidence for deep mixing of their envelopes through the interior CNO-cycle hydrogen-burning shell. Of importance in the present context is that the processing that converts $\mathrm{Ne}$ into $\mathrm{Na}$ and $\mathrm{Mg}$ into $\mathrm{Al}$ may also increase $\mathrm{He}$ at the expense of $\mathrm{H}$, so that if mixing progresses as far as the Al (Shetrone 1996a, Smith et al 1997) and Na observed enhancements indicate, the He mass fraction in the envelopes of some HB progenitor giants in M13 and $\mathrm{M} 10$, in contrast to most giants in M3 and NGC7006, might be raised sufficiently to affect the HB morphology (Langer \& Hoffman 1995, Sweigart 1997).

These results suggest that blue HBs will be generated if the precursor red giants dredge up 
large amounts of CNO-processed material in which the He mass fraction has been increased. Since classical theories of stellar evolution do not predict the existence of such deep mixing, some nonstandard mechanism must be sought, and the most promising candidate seems to be some kind of rotationally induced instability. Indirect support for this mechanism is found in the observation (Peterson 1983, Peterson et al 1995) that some HB stars in M13 exhibit rotational velocities up to $\mathrm{V} \sin \mathrm{i} \sim 40 \mathrm{~km} / \mathrm{s}$, whereas HB stars in M3 show little evidence for the existence of rotation. Further indirect evidence suggesting that He might be enhanced as a result of deep mixing comes from the study of M15 giants and HB stars. Some M15 giants exhibit striking deficiencies of $O$ and $\mathrm{Mg}$ with corresponding enhancements of $\mathrm{Na}$ and $\mathrm{Al}$ (Sneden et al 1997). At the same time, masses derived for very blue HB stars in M15 (Moehler et al 1995) are difficult to account for unless the $\mathrm{He} / \mathrm{H}$ ratio is increased to a value considered unacceptable by these authors, but which would be feasible if the atmospheres of those stars had been enriched in He by amounts consistent with the Al and Na enhancements discussed by Sneden et al (Langer et al 1997). Furthermore, if some giants mix more than others, the resulting increase in the $\mathrm{He} / \mathrm{H}$ ratio would produce a progessive "blueing" of stars in their locations along the HB. HB morphology is extremely sensitive to He abundance (Rood 1973, Dorman 1992, Sweigart 1997); variations in He mass fraction may account for the widths of HBs in clusters such as M13 (Sweigart 1997).

\section{Deep Mixing in Globular Cluster Giants vs Halo Field Giants}

A further stellar populations "anomaly" emerges when one compares the [el/Fe]-ratios of light elements in metal-poor giants of the halo field with those of globular clusters. Some degree of deep mixing surely takes place in the field giants, since the ${ }^{12} \mathrm{C} /{ }^{13} \mathrm{C}$-ratio approaches its equilibrium value with advancing evolutionary state (Sneden et al 1986). But when field and cluster giants are compared at comparable $[\mathrm{Fe} / \mathrm{H}]$ and evolutionary state, one finds no evidence either for $\mathrm{O}$ and $\mathrm{Mg}$ depletions or $\mathrm{Na}$ and $\mathrm{Al}$ enhancements such as one finds in globular clusters (Kraft 1994, Pilachowski et al 1996, Shetrone 1996a, Kraft et al 1997). Does this mean that field halo giants have, on the average, less angular momentum than cluster giants? And if some clusters have HB stars with enhanced $\mathrm{He}$ as a result of deep mixing, whereas field HB stars have no such enhancement, should not one exercise caution when comparing HB stars in clusters with HB stars in the halo field? Of special interest is the morphology of the halo field HB. It is known that the mean metallicity of the halo field is similar to that of M3, M13 and M10 (e.g. Laird et at 1993), but is the HB morphology of the field similar to one of these clusters or some combination of all three? Unfortunately, numerous selection effects (cf Kinman et al 1994) thwart attempts to establish the field morphology with accuracy sufficient to address this question.

\section{Concerns and Caveats}

\subsection{THE MG VS AL ANTICORRELATION AND DEEP MIXING}

Shetrone (1996b) demonstrated that among M13 giants in which Al abundances were very high, $\mathrm{Mg}$ was depleted at the expense not of ${ }^{25} \mathrm{Mg}$ or ${ }^{28} \mathrm{Mg}$, but rather of the ${ }^{24} \mathrm{Mg}$ isotope. If present nuclear reaction rates and giant star models are correct (Cavallo et al 1996, Langer et al 1997), production of ${ }^{27} \mathrm{Al}$ from ${ }^{24} \mathrm{Mg}$ requires a hydrogen-burning shell temperature near $70 \mathrm{MK}$, whereas the models achieve a maximum temperature of only 55MK. Langer et al (1997) suggested that hydrogen shell burning might after all be unstable (Bolton \& Eggleton 1973, von Rudloff et al 1988), thus providing episodes of elevated shell temperature; Langer et al and Dennisenkov et al (1997) also questioned the reliability of some of the nuclear reaction rates (Zaidins \& Langer 1997). Until these issues have been explored further, the need for the destruction of ${ }^{24} \mathrm{Mg}$, rather than ${ }^{25,26} \mathrm{Mg}$, remains an obstacle to full acceptance of deep mixing as a driver of the second parameter effect. Current attempts to explain the ${ }^{24} \mathrm{Mg}$ vs ${ }^{27} \mathrm{Al}$ anticorrelation as a combination of primordial and evolutionary effects appear promising (Dennisenkov et al 1997).

\section{2. "POLLUTION" ALONE IS PROBABLY NOT THE ANSWER}

On a purely primordial scenario, one might imagine that the over-abundances of $\mathrm{N}, \mathrm{Na}$ and $\mathrm{Al}$ seen in many globular cluster giants result from the pollution of the pre-existing cluster medium by 
the outpourings of massive stars. Presumably that "pre-existing" medium would ab initio reflect the abundance ratios associated with the ejecta of Type II supernovae, e.g., overabundances of $O$ and alpha elements such as $\mathrm{Mg}, \mathrm{Ca}, \mathrm{Si}$ and $\mathrm{Ti}$. But the globular cluster giants we now see are also seriously deficient in $\mathrm{O}, \mathrm{C}$ and $\mathrm{Mg}$. On a purely primordial picture, one would have to find some way to create and maintain subregions within the gas having these deficiencies until significant numbers of low mass stars could be formed from them. This requirement does not seem to have been addressed in the literature.

\subsection{WHENCE COMETH THE "EXCESS" ANGULAR MOMENTUM OF CLUSTER STARS?}

If angular momentum were conserved in shells as evolution proceeds, then the progenitors of rapidly rotating cluster $\mathrm{HB}$ stars would have to have had values of $\mathrm{V} \sin \mathrm{i}$ of nearly $100 \mathrm{~km} / \mathrm{s}$ when they were objects on the main sequence. Nothing is known of the present rotational velocities of cluster main sequence stars; field subdwarfs are known to be slow rotators (Smith 1996). Could cluster stars be spun up by stellar encounters, or perhaps by "swallowing" stellar or planetary companions as their radii increase owing to stellar evolution? Such a process must take into account an essential constraint: virtually all giants in M13 mix as they approach the red giant tip, so any mechanism advanced has to be universal in character.

\subsection{IF NOT MERIDIONAL CIRCULATION, WHAT THEN?}

Sweigart and Mengel (1979) suggested that deep mixing was driven by meridional circulation which could not take place on the first giant branch until the hydrogen-burning shell had advanced through the abundance discontinuity left behind at the close of main sequence core hydrogen burning. Although dependent on metallicity, the luminosity at which this event takes place is near the level of the HB. However, in M92 (Langer et al 1986) and NGC 6397 (Briley et al 1990) there is strong evidence that $\mathrm{C}$ is being depleted already in stars at the base of the giant branch, well before meridional circulation can be invoked as a mixing mechanism.

\section{References}

Bell, R. A., Dickens, R.J., Gustafsson, B. 1979, ApJ, 229, 604.

Bell, R. A. \& Dickens, R. 1980, ApJ, 242, 657.

Bolton, A. J. \& Eggieton, P. P. 1973, $A \mathscr{B} A, 24,429$.

Briley, M. M., Bell, R.A., Hoban, S., Dickens, R.J. 1990, ApJ, 359, 307.

Briley, M. M. \& Smith, G. H. 1993, PASP, 105, 1260.

Briley, M. M., Bell, R.A., Hesser, J.E., Smith, G.H. 1994, Can. J. Phys., 72, 772.

Briley, M. M., Smith, V.V, Suntzeff, N.B., Lambert, D.L., Bell, R.A., Hesser, J.E. 1996, Nature, $383,604$.

Brown, J. A., Wallerstein, G., Oke, J.B. 1991, AJ, 101, 1693

Buonanno, R., Buscema, G., Fusi Pecci, F., Richer, H.B., Fahlman, G.G. 1990, AJ, 100, 1811.

Carbon, D. F., Langer, G.E., Butler, D., Kraft, R.P., Trefzer, Ch., Suntzeff, N.B. $1982, A p J S, 49,207$.

Cavallo, R. M., Sweigart, A.V., Bell, R.A. 1996 ApJ, 464, L79.

Cavallo, R. M., Pilachowski, C.A., Rebolo, R. 1997 PASP, $109,226$.

Da Costa, G., Armandroft, T.E., Norris, J.E. 1992, $A J, 104,154$.

Denissenkov, P. A. \& Denissenkova, S. N. 1990, Sov. Astr. Lett., 16, 275.

Denissenkov, P. A. \& Weiss, A. 1996, A\&A, 308, 773.

Denissenkov, P. A., Weiss, A., Wagenhuber, J. 1997, $A 8 A, 320,115$.

Dickens, R., Bell, R.A., Gustafsson, B. 1979, ApJ, 232, 428.

Dorman, B. 1992, ApJS, 80, 701.

Gratton, R. G. \& Ortolani, S. 1988, $A \& A S, 73,137$.

Kinman, T. D., Suntzeff, N.B., Kraft, R.P. 1994, $A J, 108,1722$.

Kraft, R. P. 1994, PASP, 106, 553.

Kraft, R. P., Sneden, C., Langer, G.E., Prosser, C.F. 1992, $A J, 104,645$

Kraft, R. P., Sneden, C., Langer, G.E., Shetrone, M.D. 1993, $A J, 106,1490$.

Kraft, R. P., Sneden, C., Langer, G.E., Shetrone, M.D., Bolte, M. 1995, AJ, 109, 2586.

Kraft, R. P., Sneden, C., Smith, G.H., Shetrone, M.D., Langer, G.E., Pilachowski, C.A. 1997, $A J, 113,279$.

Laird, J. B. et al 1993, ASP Conf. Ser, 48, 95.

Langer, G. E., Kraft, R.P., Carbon, D.F., Friel, E., Oke, J.B. 1986, PASP, 98, 473.

Langer, G. E. \& Hoffman, R. 1995, PASP, 107, 1177.

Langer, G. E., Hofiman, R.E., Zaidins, C.S. 1997, PASP, 109, 244.

Lee, Y.-W., Demarque, P., Zinn, R. 1988, Calibration of Stellar Ages, ed. A.G.D. Philip (Schenectady, Davis), 149.

Lee, Y.-W., Demarque, P., Zinn, R. 1994, ApJ, 423, 248.

Moehler, S., Heber, U, de Boer, K.S. 1995, $A \& A, \mathbf{2 9 4}, 65$.

Norris, J. E. \& Da Costa, G. S. 1995a, ApJ, 441, L81.

Norris, J. E. \& Da Costa, G. S. 1995b, $A p J, 447,680$ 
Osborn, W. 1971, Observatory, 91, 223

Peterson, R. C. 1983, ApJ, 275, 737.

Peterson, R. C., Rood, R.T., Crocker, D.A. 1995, ApJ, 453, 214.

Pilachowski, C., Sneden, C., Kraft, R.P., Langer, G.E. 1996, $A J, 112,545$.

Preston, G. W., Schectman, S.A., Beers, T.C. 1991, ApJ, 375, 121.

Richer, H. B. et al 1996, $A p J, 463,602$.

Rood, R. 1973, ApJ, 184, 815.

Sandage, A. R. \& Wildey, R. 1967, ApJ, 150, 469.

Searle, L. \& Zinn, R. 1978, ApJ, 225, 357.

Shetrone, M. D. $1996 \mathrm{a}, A J, 112,1517$.

Shetrone, M. D. 1996b, $A J, 112,2639$.

Smith, G. H., Shetrone, M.D., Bell, R.A., Churchill, C.W., Briley, M.M. 1996, AJ, $112,1511$.

Smith, G. H., Shetrone, M.D., Briley, M.M., Churchill, C.W., Bell, R.A. 1997, PASP, $109,236$.

Smith, G. H., Kraft, R.P., Sneden, C., Shetrone, M.D., Fullbright, J. 1997, AJ, (submitted).

Smith, V. V. 1996, private comm.

Sneder, C., Pilachowski, C.A., Vandenberg, D.A. 1986, ApJ, 311, 826.

Sneder, C., Kraft, R.P., Prosser, C.F., Langer, G.E. 1992, AJ, 104, 2121.

Sneden, C., Kraft, R.P., Langer, G.E., Prosser, C.F., Shetrone, M.D. 1994, AJ, 107, 1773.

Sneden, C., Kraft, R.P., Shetrone, M.D., Smith, G.H., Laner, G.E., Prosser, C. 1997, $A J$, (in press).

Stetson, P. B., Hesser, J.E., Smith, G.H., Vandenberg, D.A., Bolte, M. 1989, AJ, 97, 1360.

Stetson, P. B., Vandenberg, D.A., Bolte, M. 1996, PASP, 108, 560.

Suntzeff, N. B. 1993, ASP Conf. Ser. 48, 167.

Sweigart, A. V. 1997, ApJ, 474, L23.

Sweigart, A. V. \& Mengel, J. G. 1979, ApJ, 229, 624.

Trefzger, C. F., Langer, G.E., Carbon, D.F., Suntzeff, N.B., Kraft, R.P. 1983, ApJ, $266,144$.

van den Bergh, S. 1967, $A J, 72,70$.

von Rudloff, I. R., Vandenberg, D.A., Hartwick, F.D.A. 1988, ApJ, 324, 840.

Zaidins, C. \& Langer, G. E. 1997, PASP, 109, 252.

Zinn, R. 1973, ApJ, 182, 183.

Zinn, R. 1993, ASP Conf. Ser. 48,38 . 